\title{
UNA TIPOLOGÍA ANALÍTICA DE LAS REDES DE APOYO SOCIAL EN INMIGRANTES AFRICANOS EN ANDALUCÍA
}

\author{
Manuel F. Martínez García, Manuel García Ramírez \\ e Isidro Maya Jariego \\ Universidad de Sevilla \\ E-mail: fgarcia@cica.es
}

\begin{abstract}
RESUMEN
Elaboramos, por medio del análisis de conglomerados, una clasificación de las redes de apoyo social de los inmigrantes africanos afincados en Andalucía. Como variables criterio, utilizamos diversos indicadores del tamaño, la composición, la disponibilidad, la utilización y la suficiencia de la estructura personal de ayuda. Con un colectivo de 600 africanos, obtuvimos cuatro categorías: 1) redes pequeñas con predominio de amigos compatriotas; 2) redes mínimas con predominio de amigos compatriotas; 3) redes mixtas de tamaño medio centradas en los amigos, y 4) redes de tamaño medio centradas en la familia. La tipología distingue significativamente los niveles de bienestar psicológico, el estilo de utilización de servicios y otras características psicológicas, sociales y demográficas.
\end{abstract}

El análisis de las redes sociales tiene una larga tradición de estudio en el ámbito de las migraciones internacionales. Desde que en 1964 los demógrafos australianos MacDonald y MacDonald introdujeron el concepto de cadena migratoria, se ha desarrollado profusamente una línea de investigación sobre la composición, dirección y persistencia de los flujos migratorios, que ha utilizado como referente teórico el concepto de redes sociales (Boyd, 1989). Frente a la consideración inerte y pasiva del migrante, típica de los modelos push/pull, la introducción del concepto cadena migratoria supuso un cambio significativo en el análisis de las migraciones, ya que dotó al sujeto de un papel activo en 
dicho proceso, capaz de formular estrategias de supervivencia y readaptación en contextos de cambios macroestructurales (Ciafardo, 1991). Esta capacidad de gestión del proceso migratorio por parte de la red de contactos interpersonales es el antecedente directo de otros trabajos, como los de Mitchel (1969), que comienzan a generalizar al ámbito de la emigración el concepto de red, propuesto en los estudios antropológicos pioneros de Barnes y Bott en la década de los cincuenta (Bott, 1990; Gregorio, 1998; Requena, 1991, 1994).

Los contactos interpersonales adquieren un papel esencial tanto para explicar la decisión última de emigrar (Sycip y Fawcet, 1988) como en el propio proceso de adaptación en la nueva sociedad, atribuyendo, incluso, papeles diferenciales a las interacciones más personales, frecuentes e intensas (lazos fuertes, vinculados a la esfera psicológica), de aquellas que se sitúan en el polo opuesto y que posibilitan un mejor conocimiento del entorno (Granovetter, 1973).

Con carácter general, la red social es un término usado por los científicos sociales para abarcar la comprensión de las diversas dinámicas interpersonales que tienen lugar en el entorno inmediato de los seres humanos (Litwin, 1995). Si tenemos en cuenta que las redes reflejan un rango de relaciones que las personas mantienen con los otros, en este trabajo nos acercaremos a las mismas desde una perspectiva psicológica, teniendo en cuenta sólo a aquellos elementos de las mismas (conformando un subconjunto) que son percibidos por los sujetos como potenciales proveedores de ayuda. Esta perspectiva se enmarca dentro de lo que conocemos en Psicología como apoyo social y tuvo sus raíces en la filosofía del Movimiento de Salud Mental Comunitaria en la década de los sesenta al plantear, como alternativa a la institucionalización, tratamientos y cuidados basados en un mayor protagonismo de los recursos sociales y comunitarios. El despegue del área como foco de interés científico ocurre a partir de los años setenta con trabajos empíricos y sistemáticos sobre apoyo social en los que se constató, como patrón de búsqueda de ayuda, que las personas con problemas emocionales o en período de crisis acuden antes a su entorno próximo que a los servicios profesionales de salud mental (psicólogos, psiquiatras, etc.).

Los primeros trabajos de interés son los de Cassel (1974) y Cob (1976), sobre la variabilidad que establecen nichos ecológicos diferentes en el afrontamiento de experiencias estresantes; y los de Caplan (1974), donde se describen los aspectos positivos del apoyo sobre la salud. En este contexto, Caplan definió los sistemas de apoyo como «agregados sociales continuos que dotan al individuo de oportunidades de obtener feedback sobre sí mismo y la validación de sus expectativas sobre los demás»(Caplan, 1974, p. 4). En la actualidad, el apoyo social es un tópico ampliamente difundido en la literatura psicológica y es entendido tanto como factor etiológico (del bienestar y calidad de vida o de desórdenes y problemas) como estrategia interventiva (al objeto de optimizar los aspectos positivos de las interacciones sociales), o como constructo relacional (valor heurístico) para comprender relaciones entre la dimensión psicosocial y los procesos tanto de salud-bienestar como de enfermedad/problemas.

En el campo de las migraciones existe una abundante literatura que vincula 
las redes de apoyo social e integración (Lynam, 1985; Die y Seelbach, 1991; Leslie, 1992; Aroian, 1992; Berry, 1997; Clarke y Jensen, 1997; Martínez, García y Maya, 1999). Son diversas las funciones atribuidas al sistema de apoyo social en el proceso migratorio: 1) contribuye a la satisfacción de necesidades; 2) favorece la participación social; 3) es un marco de referencia para los procesos de comparación social y evaluación de la realidad; 4) favorece la ocurrencia de eventos positivos y ayuda a afrontar los negativos, y 5) potencia la capacidad de adaptación favoreciendo la integración social.

Las redes de apoyo social de los inmigrantes se han descrito habitualmente por medio del análisis de dimensiones morfológicas y funcionales, tales como el tamaño, la multiplicidad, la composición, la suficiencia, etc. (Furnham y Li, 1993; Golding y Baezconde-Garbanati, 1990; Griffith y Villavicencio, 1985; Janes, 1990; Kuo y Tsai, 1986; Miller-Loncar et al., 1998; Sluzki, 1992; Vega et al., 1991). En esta línea hemos analizado en trabajos anteriores las redes de apoyo de inmigrantes africanos y latinoamericanos asentados en Andalucía (Martínez, García, Maya, Rodríguez y Checa, 1996; Martínez et al., 1999). De acuerdo con los datos, parece que la estructura colectiva de la comunidad inmigrada en el lugar de destino condiciona las posibilidades de constitución, composición y crecimiento del círculo personal de apoyo: es decir, la naturaleza reciente del fenómeno contribuye a que la población foránea disponga de redes de ayuda instrumental y afectiva más pequeñas que la población nativa, y, de este modo, acentúa uno de los rasgos propios del entorno de los recién llegados. Además, esta disminución en la disponibilidad de recursos sociales naturales afecta tanto a la composición como a la multiplicidad de los proveedores de ayuda: por ejemplo, es común que los amigos sustituyan temporalmente a la familia, y que se observe una relación inversa entre la polivalencia de los parientes y la de los amigos. En ese contexto, los inmigrantes recurren a cuatro estrategias de reconstrucción de la red: la incorporación de españoles, el reagrupamiento familiar, la concentración de funciones en unos pocos vínculos, y la utilización de grupos — en lugar de individuos- como unidades de apoyo. Estos resultados coinciden, en líneas generales, con los antecedentes de la literatura sobre apoyo social e inmigración ya referenciada.

En contraste, ha sido menos usual la construcción de tipologías analíticas basadas en las diversas combinaciones posibles de las categorías antes reseñadas. La finalidad de la construcción de tipologías es constatar covarianzas sistemáticas en los agrupamientos de las características de la red (Litwin, 1997) que, al integrar diversas dimensiones, permite advertir estructuras permanentes más allá de los cambios a los que están sujetos los indicadores singulares. Desde los trabajos iniciales de Adams (1986), un paso significativo en esta dirección se deriva de los estudios de Wenger y colaboradores realizados sobre personas mayores en Wales, en los que correlacionaba los distintos tipos de red social con los cambios en la propia estructura reticular (Wenger y Shahtahmasebi, 1991), la autoayuda y la ayuda mutua (Wenger, 1993), la vulnerabilidad (Gordon y Wenger, 1993) o los factores de riesgo (Wenger, 1997). La tipología de redes encontrada se fundamentada en tres factores básicos: 1) la disponibili- 
dad de familiares cercanos en el ámbito local; 2) el nivel de implicación de la familia, los amigos y los vecinos, y 3) el nivel de interacción con la comunidad y con grupos voluntarios (Wenger, 1991). Se describieron cinco tipos de redes, de los que uno de ellos era característico de individuos que habían emigrado: concretamente, la «red centrada en vínculos con la comunidad extensa». Ello refleja el contexto interpersonal de los ancianos de clase media retirados, que mantienen un gran número de lazos con amigos, vecinos y organizaciones voluntarias, pero que - al cambiar su lugar de residencia- no cuentan con parientes en el entorno local. A pesar de que esta autora refiere un tipo de red característico de los individuos desplazados, la tipología se aplica a la población de ancianos en contextos familiares normativos, y es de suponer que los inmigrantes difieren en cierta medida en las modalidades de sistemas de apoyo que están en condiciones de conformar.

Otra referencia obligada es el estudio de Litwin (1995), en el que describe una tipología analítica de las redes de 259 ancianos rusos inmigrantes en Israel. En trabajos posteriores, utilizando esa técnica taxonómica, relaciona los distintos tipos de redes con la utilización de servicios de salud (1997), con patrones conductuales de dar y recibir ayuda (Litwin, 1999), etc. Utilizando la técnica estadística de los conglomerados, analiza seis variables estructurales y de interacción: tamaño de la red, porcentaje de lazos íntimos, composición, duración de los lazos, frecuencia de contactos y proximidad residencial. Como resultado obtiene cuatro categorías fundamentales, en las que la dimensión determinante fue la composición: 1) redes de familia nuclear, compuestas casi completamente por el esposo y los hijos; 2) redes con miembros de la familia extensa —además de los vínculos de la familia nuclear, y una representación modesta de amigos-; 3) redes centradas en sus dos terceras partes en los amigos, y 4) redes de lazos difusos, de composición muy diversificada.

Del mismo modo, constató que estas cuatro categorías diferían en su capacidad de ayuda, de modo que las redes familiares proporcionan más apoyo psicológico que las no familiares (o parcialmente familiares). Además, según este autor, puede intuirse un patrón de desarrollo de la red entre los ancianos inmigrantes, que evoluciona desde la conformación inicial de redes de familiares cercanos a una expansión progresiva, que incluye un mayor rango de vínculos.

El análisis de redes de apoyo social se configura, por tanto, como un instrumento eficaz de investigación con poblaciones en transición ecológica. Permite conocer los procesos a través de los cuales las personas van consolidando su competencia psicosocial para atender sus necesidades y hacer frente a las demandas ambientales, favoreciendo la adaptación al entorno, participar activamente en él y vivir satisfactoriamente. En este trabajo pretendemos: 1) clasificar los tipos de redes de apoyo social que desarrollan un colectivo de inmigrantes africanos en Andalucía, valiéndonos de dimensiones estructurales y funcionales, y 2) determinar las diferencias entre esas categorías en función de la multiplicidad y satisfacción con los vínculos de la red, la utilización de servicios formales y el bienestar psicológico. 


\section{MÉTODO}

\section{PARTICIPANTES}

La población de nuestra encuesta estuvo compuesta por los inmigrantes africanos de 16 o más años residentes en cinco provincias andaluzas: Málaga, Almería, Granada, Sevilla y Cádiz. Dada la imposibilidad de utilizar un método aleatorio, se efectuó un muestreo por cuotas, estratificado por provincias con afijación proporcional. Las cuotas se establecieron en función de las variables de nacionalidad y sexo, y se basaron en la distribución de inmigrantes africanos regularizados (Izquierdo, 1995).

Se determinó un grupo de 600 personas y un nivel de confianza de 95,5 por 100. De acuerdo con los parámetros establecidos, el error que afecta a nuestro estudio asciende a 1,99. Sin embargo, teniendo en cuenta que el error de un muestreo por cuotas es igual al error de una muestra aleatoria de igual tamaño incrementado en un 50 por 100 (Moser, 1973), el error quedaría establecido en 2,99.

Para la selección de los sujetos se utilizaron dos estrategias complementarias: la técnica de «bola de nieve» (87,4 por 100 de los entrevistados) y el contacto con instituciones que proporcionan servicios a los inmigrantes (12,6 por 100). La selección en cadena supone un sesgo hacia individuos que comparten características estructurales (Requena, 1996; Rodríguez, 1995), y es una limitación adicional en la representatividad del colectivo estudiado. Por ello se utilizó, como vía alternativa, el reclutamiento a través de organizaciones sociales, así como una "guía de estudio" para garantizar la heterogeneidad de los entrevistados (Taft, 1986).

Los rechazos a responder el cuestionario no alcanzaron el 4 por 100 del total de contactos. La selección se ajustó fielmente a las cuotas preestablecidas. Sólo hubo modificaciones en las cuotas femeninas de dos de las nacionalidades más minoritarias, que fueron compensadas con entrevistas a hombres del mismo país de procedencia, y algún reajuste entre provincias de la población argelina.

\section{Características sociodemográficas de los entrevistados}

Las características sociodemográficas de los entrevistados se describen en la tabla 1. La media de edad de los participantes se sitúa en 31,2 años, no habiendo diferencias significativas entre sexos. Si más de la mitad de los entrevistados afirma haber realizado estudios secundarios o superiores, el nivel de estudios es algo menor entre las mujeres. La juventud del colectivo se relaciona con el carácter reciente del fenómeno migratorio ya que más de dos tercios de los africanos (68,34 por 100) llevan en España menos de cinco años.

Por su parte, hay claras diferencias en la distribución de hombres y mujeres en las distintas categorías del estado civil: mientras entre los hombres predominan ligeramente los solteros, entre las mujeres el grupo más numeroso es el de las que viven en pareja - ya sea en matrimonio o sin formalizar la relación-. Pero quizá 
la diferencia más característica es la elevada proporción de mujeres divorciadas, separadas o viudas (22,32 por 100) en comparación con los hombres.

Por último, en el momento de la entrevista no tenía completamente en regla su situación administrativa en España un cuarto del colectivo estudiado. La proporción de irregulares es menor entre mujeres. La generalidad de los entrevistados $(74,5$ por 100$)$ afirma haber entrado en nuestro país con visado de turista, o sólo con el pasaporte cuando no era necesario el primero. Son minoritarios los que entraron con visado de estudiante $(8,7$ por 100$)$, clandestinamente $(8,6$ por 100$)$ o con contrato de trabajo (1,3 por 100$)$.

\section{TABLA 1}

Caracteristicas sociodemográficas

\begin{tabular}{|c|c|c|c|c|c|}
\hline & $F$ & $\%$ & & $F$ & $\%$ \\
\hline \multicolumn{6}{|l|}{ Estado civil } \\
\hline Soltero ............ & 260 & 43,40 & Hombres ....... & 385 & 64,20 \\
\hline Casado/Pareja ........................... & 286 & 47,74 & 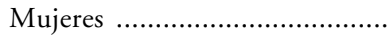 & 215 & 35,80 \\
\hline Divorciado, separado, viudo ...... & 53 & 8,84 & & & \\
\hline \multicolumn{3}{|l|}{ Edad } & Tiempo estancia & 9 & 1617 \\
\hline$(26-35)$ & 332 & 55,33 & 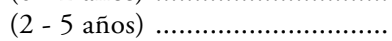 & 31 & 52,17 \\
\hline 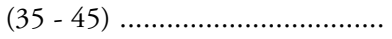 & 106 & 17,67 & 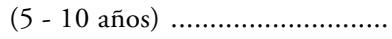 & 123 & 20,50 \\
\hline$(>45)$ & 32 & 5,33 & (> 10 años) & 67 & 11,16 \\
\hline \multicolumn{3}{|l|}{ Estudios } & Docume & & \\
\hline 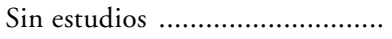 & 100 & 16,75 & Permiso residencia .................... & 144 & 24,04 \\
\hline Primarios ..................................... & 184 & 30,82 & Permiso trabajo .......................... & 290 & 48,41 \\
\hline Secundarios $\ldots \ldots \ldots \ldots \ldots \ldots \ldots \ldots \ldots \ldots \ldots \ldots \ldots \ldots \ldots \ldots \ldots$ & 168 & 28,14 & 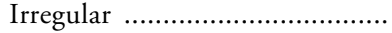 & 80 & 13,35 \\
\hline \multirow[t]{2}{*}{ 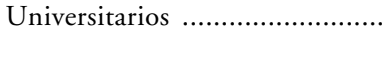 } & 145 & 24,29 & En trámite & 70 & 11,68 \\
\hline & & & Otra & 15 & 2,50 \\
\hline
\end{tabular}

FUENTE: Encuesta sobre la integración social de los inmigrantes africanos en Andalucía, 1995. Elaboración propia.

\section{INSTRUMENTOS}

Entrevista estructurada sobre proceso migratorio, necesidades, recursos y problemas. El cuestionario constó de 42 preguntas, estructuradas en siete bloques temáticos: datos sociodemográficos y legales, proyecto migratorio, indicadores de aculturación, percepción de problemas, percepción de rechazo, utilización de recursos y satisfacción vital. Para la elaboración del cuestionario se revisó literatura científica relevante (v.g., IOÉ, 1987; Izquierdo, 1994; Giménez, 1992; etcétera) y se realizó un estudio exploratorio con 23 entrevistas en profundidad a inmigrantes e informantes clave. En el cuestionario, algunos aspectos fueron medidos con un formato de pregunta cerrada en el que las contestaciones se graduaron en una escala de intensidad. En concreto, la evaluación de 
problemas que preocupan actualmente (nada, algo, bastante, mucho) y la presencia de problemas en las distintas áreas de necesidad (verdadero-falso), con los que obtuvimos unos coeficientes de fiabilidad de 0,85 y 0,70, respectivamente.

Entrevista semiestructurada de apoyo social. Se utilizó la Arizona Social Support Interview Schedule (ASSIS) (Barrera, 1980), que explora seis categorías de apoyo: sentimientos personales, ayuda material, consejo, feedback positivo, asistencia física y participación social. Dicha categorización recoge de manera exhaustiva los tipos de apoyo social que tradicionalmente se han definido en la literatura especializada: el Apoyo Emocional se describe por la combinación de las áreas de Sentimientos Personales y Participación Social, el Apoyo Informacional por las categorías de Consejo y Feedback positivo, y el Apoyo Tangible por la Ayuda Física y Material.

Esta entrevista facilita a los sujetos la discriminación de qué se entiende por acción de ayuda, quién la provee y cuál es su naturaleza. Además, se obtienen cuatro indicadores acerca de la estructura y funcionalidad del Sistema de Apoyo: tamaño percibido de la red, necesidad de apoyo, utilización de la red para obtenerlo y satisfacción con el apoyo recibido.

El tamaño percibido de la red hace referencia al número de personas disponibles para proveer apoyo al menos en una de las categorías citadas y permite conocer la procedencia de los vínculos, la disponibilidad de la red para cada uno de los tipos de apoyo y la multiplicidad, atributo que hace referencia al potencial sinérgico de los lazos sociales. Por todo ello, el ASSIS puede ser considerado como un potente instrumento para medir la estructura y las características de los recursos naturales de apoyo o, en otras palabras, el Sistema de Apoyo Social (Thoits, 1982). En cada una de dichas dimensiones se obtuvo un indicador adecuado de consistencia interna: el coeficiente de Cronbach fue 0,85 en disponibilidad, 0,73 en utilización, 0,62 en satisfacción y 0,73 en necesidad.

A partir de la traducción, adaptación y pretest realizado con un colectivo de inmigrantes, se han introducido modificaciones para tomar indicadores de la suficiencia percibida de la red de apoyo, la estabilidad percibida de la red de apoyo y las razones de no interacción con determinados vínculos. Además, se ha incluido información sobre la ubicación actual del vínculo, su condición de inmigrante o no, etcétera. Tales modificaciones no afectan a la estructura global de la entrevista original, sino que suponen básicamente la inclusión de información complementaria.

\section{Procedimiento}

La encuesta fue realizada por un total de 13 entrevistadores, divididos en dos grupos, en cada uno de los cuales un miembro del equipo de investigación actuaba como supervisor. Los encuestadores fueron licenciados estudiantes de posgrado (en las áreas de psicología social y antropología social), en la mayoría de los casos con experiencia previa de entrevistas. Un pequeño grupo de cuatro encuestadores realizó el grueso de las entrevistas (89 por 100), garantizando la homogeneidad del proceso de aplicación.

La duración de la entrevista osciló entre una hora y una hora y media aproxi- 
madamente. Se comenzaba siempre en español y, en el caso de encontrar problemas de comprensión, se continuó con el idioma de elección del entrevistado. Participaron siete inmigrantes de distintas nacionalidades — marroquíes, argelinos y senegaleses - que desempeñaron labores de intérprete y actuaron como mediadores que facilitaron la entrada y los contactos en cada comunidad. En la mayoría de los casos fueron del mismo sexo y cultura que los entrevistados, y algunos tenían experiencia en actividades de recogida de información.

\section{ANÁLISIS DE RESULTADOS}

\section{DESCRIPCIÓN Y ANÁLISIS DE LOS TIPOS DE RED}

Se aplicó el procedimiento Quick Cluster para agrupar los casos en cuatro agregados. Se seleccionaron 12 características estructurales y funcionales de las redes de apoyo referidas a las dimensiones de tamaño, composición, disponibilidad, utilización y suficiencia. La clasificación final por tipo de red incluyó 572 casos, mientras que 28 se excluyeron del análisis debido a datos missing en las variables criterio.

Las dos dimensiones principales que parecen determinar el tipo de estructura reticular son el tamaño y la composición de la red (gráfico 1). El tamaño medio de las redes para el total de los entrevistados es de 3,75 y dista mucho del número de vínculos disponibles para la población general, que oscila, según diversos estudios e instrumentos de evaluación, entre 8 y 10 personas aproximadamente (Golding y Baezconde-Garbanati, 1990; Gottlieb, 1981; Guimón et al., 1985; Hammer et al., 1978; McFarlane et al., 1980). Sin embargo, el tamaño varía en las diferentes tipologías analizadas, de modo que podemos distinguir redes medianas, formadas por seis o siete personas; pequeñas, de unas cuatro personas, y tan escasas que no llegan a los dos contactos de promedio. De esta forma, podemos distinguir: Tipo 1, redes de tamaño medio centradas en la familia; Tipo 2, redes mixtas de tamaño medio centradas en los amigos (españoles y connacionales); Tipo 3, redes mínimas con predominio de amigos compatriotas; y Tipo 4, redes pequeñas con predominio de amigos compatriotas. La tabla 2 resume los datos de las variables criterio y las diferencias significativas (estadístico F) entre las cuatro categorías.

Las dos primeras categorías agrupan en torno al 14 por 100 de inmigrantes que han desarrollado redes más amplias y, en consecuencia, son útiles para analizar las características psicosociales de aquellos inmigrantes que han tenido más éxito en la reconstrucción de su entorno social. Por un lado, un porcentaje pequeño $(6,12$ por 100) recurre prioritariamente a familiares (86 por 100) y apenas cuenta con españoles en su red, mientras que un segundo grupo (7,87 por 100) centra sus demandas de apoyo en los amigos — que llegan a suponer el 92 por 100 de los vínculos - y tiene un entorno personal cercano bien equilibrado entre compatriotas (67 por 100) y miembros de la sociedad de acogida (43 por 100).

La tercera categoría agrupa a un tercio de los encuestados que cuentan sólo con 1,71 personas de media en su círculo de apoyo, lo que, de acuerdo con el 
autoinforme de los sujetos, resulta insuficiente para satisfacer las necesidades de la persona focal. Por último, el mayor número de inmigrantes $(53,67$ por 100) cuenta con redes pequeñas — de unas cuatro personas, mayoritariamente del mismo origen nacional (71,7 por 100)—, que están compuestas en sus dos terceras partes por amigos.

\section{GRÁFICO 1}

Tipología de las redes de apoyo de los inmigrantes africanos en Andalucía

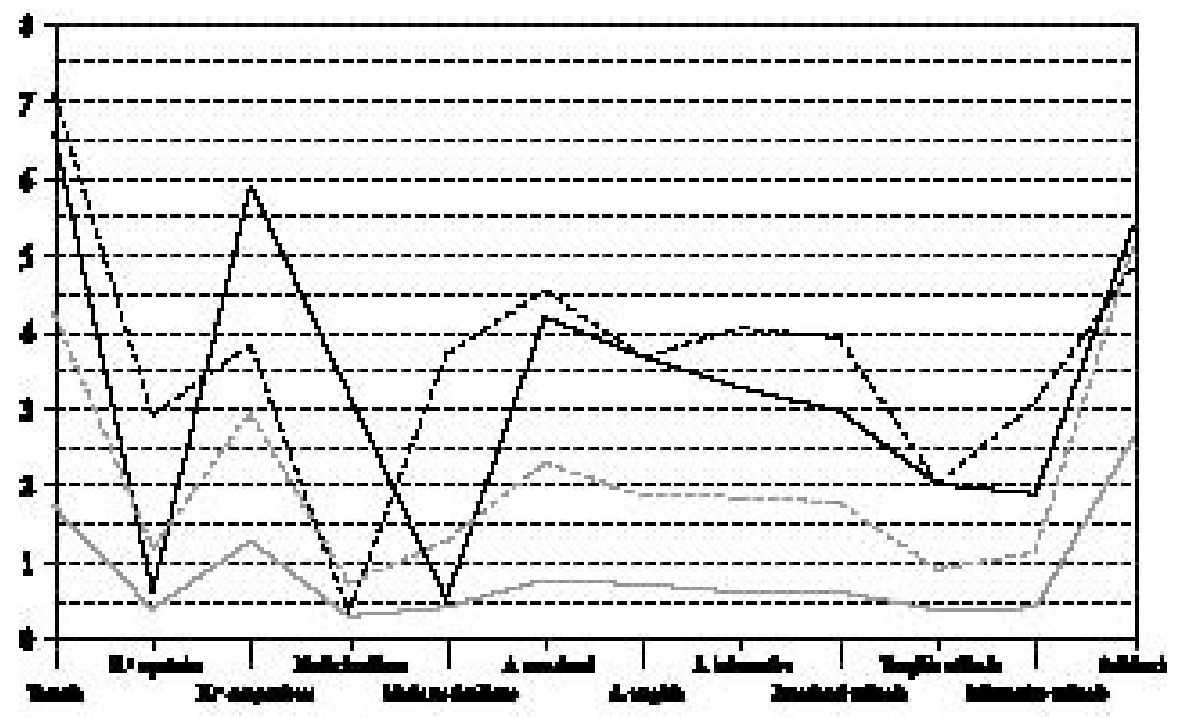

Tpe 1 - - Tpo2

Las diferencias significativas en apoyo social vienen determinadas básicamente a partir de los bajos niveles observados en la categoría Tipo 3, tanto para el apoyo disponible, utilizado como suficiente. Igualmente se observan diferencias entre los dos subgrupos con redes de seis o siete vínculos: esto es, los inmigrantes con redes mixtas centradas en los amigos disponen de más recursos informativos y utilizan más información y apoyo emocional de su entorno que aquellos con redes centradas en la familia. Ambos tipos, con contactos por encima de la media, son singulares además por su composición: si en un caso está tan orientada hacia los parientes que la media de no familiares es equiparable a la de las redes más pequeñas, en otro caso el sesgo hacia los iguales se refleja en una media de familiares tan reducida como la de las redes mínimas. 


\section{TABLA 2}

Medias y análisis oneway de la varianza de las variables de apoyo por tipo de red

\begin{tabular}{|c|c|c|c|c|c|c|c|c|c|}
\hline & \multicolumn{8}{|c|}{ Tipo de red de apoyo } & \multirow[b]{3}{*}{$F$} \\
\hline & \multicolumn{2}{|c|}{ Tipo 1} & \multicolumn{2}{|c|}{ Tipo 2} & \multicolumn{2}{|c|}{ Tipo 3} & \multicolumn{2}{|c|}{ Tipo 4} & \\
\hline & $\bar{x}$ & $S D$ & $\bar{x}$ & $S D$ & $\bar{x}$ & $S D$ & $\bar{x}$ & $S D$ & \\
\hline \multicolumn{10}{|l|}{ Tamaño } \\
\hline Tamaño de la red de apoyo .................................... & 6,57 & 2,43 & 7,09 & 2,49 & 1,71 & 1,28 & 4,23 & 1,71 & $183,8^{* * *}$ \\
\hline \multicolumn{10}{|l|}{ Composición } \\
\hline Número de españoles en la red de apoyo ........... & 0,60 & 0,95 & 2,91 & 2,38 & 0,38 & 0,71 & 1,16 & 1,32 & $51,6^{* * *}$ \\
\hline Número de compatriotas en la red de apoyo ...... & 5,89 & 2,64 & 3,82 & 2,10 & 1,26 & 1,16 & 2,93 & 1,55 & $108,8^{* * *}$ \\
\hline Media de familiares por tipo de apoyo ................... & 3,20 & 1,33 & 0,32 & 0,54 & 0,28 & 0,38 & 0,71 & 0,69 & $197,2^{* * *}$ \\
\hline Media de no familiares por tipo de apoyo ........... & 0,50 & 0,74 & 3,70 & 1,03 & 0,40 & 0,42 & 1,26 & 0,77 & $274,1^{* * *}$ \\
\hline \multicolumn{10}{|l|}{ Disponibilidad } \\
\hline 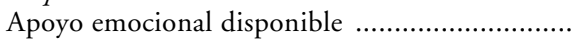 & 4,18 & 1,20 & 4,53 & 1,72 & 0,76 & 0,65 & 2,28 & 0,75 & $330,7^{* * *}$ \\
\hline 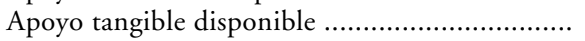 & 3,67 & 1,95 & 3,67 & 1,35 & 0,70 & 0,63 & 1,84 & 0,84 & $179,5^{* * *}$ \\
\hline Apoyo informativo disponible ............................. & 3,25 & 1,50 & 4,04 & 1,35 & 0,60 & 0,52 & 1,83 & 0,86 & $243,3^{* * *}$ \\
\hline \multicolumn{10}{|l|}{ Utilización } \\
\hline Apoyo emocional utilizado .................................. & 2,95 & 1,45 & 3,92 & 2,01 & 0,60 & 0,55 & 1,76 & 0,86 & $175,6^{* * *}$ \\
\hline 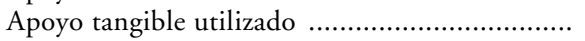 & 2,00 & 2,04 & 2,01 & 1,48 & 0,37 & 0,47 & 0,89 & 0,81 & $58,5^{* * *}$ \\
\hline Apoyo informativo utilizado .............................. & 1,87 & 1,80 & 3,07 & 1,49 & 0,39 & 0,45 & 1,11 & 0,80 & $123,3^{* * *}$ \\
\hline \multicolumn{10}{|l|}{ Suficiencia } \\
\hline Número de tipos de apoyo suficientes .................. & 5,37 & 1,08 & 4,84 & 1,63 & 2,61 & 2,18 & 5,09 & 1,32 & $92,85^{* * *}$ \\
\hline
\end{tabular}

\section{*** $\mathrm{p}<0,001$.}

FUENTE: Encuesta sobre la integración social de los inmigrantes africanos en Andalucía, 1995. Elaboración propia. 


\section{SATISFACCIÓN Y MULTIPLICIDAD DEL APOYO POR TIPO DE RED}

En la tabla 3 se presentan los resultados de satisfacción con los intercambios de apoyo y multiplicidad de los diferentes vínculos en función de la tipología de redes. De acuerdo con los datos, constatamos que se observan diferencias en la satisfacción con los intercambios de apoyo: los individuos con red menos extensa (Tipo 3) se muestran menos complacidos que el resto respecto a las distintas categorías de apoyo, con excepción de la ayuda material, en la que no se encontraron diferencias entre los cuatro grupos.

Los vínculos de los individuos con redes centradas en la familia muestran un patrón de multiplicidad bien diferenciado del resto de tipos de red: los padres, hermanos e hijos proporcionan más clases de ayuda que en los demás clusters, mientras que los amigos cumplen menos funciones que en las redes donde predominan los contactos basados en la amistad (Tipos 2 y 4), hasta el punto de que su capacidad de proveer recursos diversos se encuentra en niveles tan bajos como los de las estructuras mínimas (Tipo 3).

\section{CADENAS MIGRATORIAS Y TIPO DE RED}

La naturaleza de la cadena migratoria en la que el inmigrante se ubica está directamente relacionada con el tipo de red de apoyo de que dispone en el nuevo contexto. La clase de estructura reticular de ayuda está vinculada al número de familiares residentes en España, al número de emigrantes en la familia y al lugar que ocupa la persona focal en el desarrollo de la serie migratoria familiar.

Los extranjeros que concentran las demandas de protección y asistencia en la familia tienen una media de 5,22 parientes en España, mientras que en los demás conglomerados no se alcanzan los dos familiares como promedio (tabla 4). De acuerdo con ello, el grupo donde es más probable contar con tres o más emigrantes en la familia —además de que éstos residan en España- es el Tipo 1. En sentido contrario — pero también de forma significativa-, en torno a un tercio de quienes disponen de redes mínimas no tienen otros emigrantes en la familia, mientras que el 25 por 100 cuenta a lo sumo con uno. Asimismo, es consistente con estas tendencias el hecho de que aquellos con pequeñas redes de compatriotas tengan el mayor índice de antecedentes migratorios en la familia, contando un porcentaje significativo de ellos con dos allegados que han emigrado.

Como cabría esperar, casi un tercio de los individuos que cuentan con la familia como fuente prioritaria de ayuda consideran consumada la reagrupación y son, en consecuencia, el grupo que significativamente contesta en mayor proporción que su familia "ya está aquí». 


\section{TABLA 3}

Medias y análisis oneway de la varianza de multiplicidad y satisfacción por tipo de red

Tipo de red de apoyo

\begin{tabular}{|c|c|c|c|c|c|c|c|c|c|}
\hline & \multirow{2}{*}{\multicolumn{2}{|c|}{ Tipo 1}} & & & & & & & \\
\hline & & & \multicolumn{2}{|c|}{ Tipo 2} & \multicolumn{2}{|c|}{ Tipo 3} & \multicolumn{2}{|c|}{ Tipo 4} & \multirow[b]{2}{*}{$F$} \\
\hline & $\bar{x}$ & $S D$ & $\bar{x}$ & $S D$ & $\bar{x}$ & $S D$ & $\bar{x}$ & $S D$ & \\
\hline \multicolumn{10}{|l|}{ Satisfacción } \\
\hline Con la expresión de sentimientos ....................... & 51,5 & 30,8 & 56,8 & 23,7 & 38,0 & 33,2 & 51,4 & 27,7 & $8,29^{* * *}$ \\
\hline Con la ayuda material ......................................... & 32,0 & 33,7 & 36,2 & 34,9 & 28,5 & 33,4 & 37,1 & 33,9 & 2,06 \\
\hline Con el consejo & 34,0 & 34,1 & 50,4 & 30,1 & 27,7 & 33,5 & 40,9 & 32,5 & $7,55^{* * *}$ \\
\hline Con el feedback positivo .................................. & 41,8 & 32,9 & 56,9 & 24,2 & 33,7 & 33,4 & 45,9 & 30,3 & $7,99^{* * *}$ \\
\hline 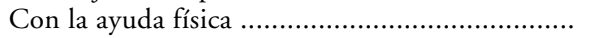 & 42,8 & 33,5 & 42,9 & 31,4 & 25,9 & 32,6 & 37,3 & 32,8 & $5,52^{* * *}$ \\
\hline Con la participación social ................................ & 62,3 & 20,1 & 61,3 & 20,5 & 31,2 & 31,2 & 22,2 & 22,2 & $9,86^{* * *}$ \\
\hline \multicolumn{10}{|l|}{ Multiplicidad } \\
\hline 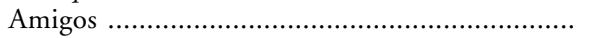 & 1,71 & 2,06 & 5,24 & 0,93 & 1,67 & 2,01 & 3,81 & 2,11 & $66,53^{* * *}$ \\
\hline 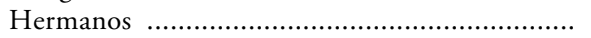 & 2,60 & 2,63 & 0,06 & 0,25 & 0,22 & 0,93 & 0,82 & 1,66 & $28,86^{* * *}$ \\
\hline 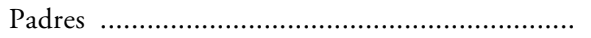 & 2,08 & 2,48 & 0,26 & 0,68 & 0,19 & 0,55 & 0,55 & 1,16 & $29,26^{* * *}$ \\
\hline 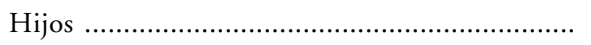 & 1,37 & 2,03 & 0,17 & 0,61 & 0,12 & 0,69 & 0,24 & 0,90 & $17,94^{* * *}$ \\
\hline 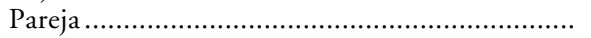 & 2,42 & 2,44 & 0,84 & 0,70 & 0,85 & 1,63 & 1,47 & 2,09 & $08,69^{* * *}$ \\
\hline
\end{tabular}

Satisfacción

Con la expresión de sentimientos

Con la ayuda material

Con el consejo

Con el feedback positivo

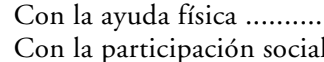

\section{Multiplicidad}

*** $\mathrm{p}<0,001$.

FUENTE: Encuesta sobre la integración social de los inmigrantes africanos en Andalucía, 1995. Elaboración propia. 
TABLA 4

Caracteristicas de las cadenas migratorias por tipo de red

\begin{tabular}{|c|c|c|c|c|c|c|c|c|c|}
\hline & \multicolumn{8}{|c|}{ Tipo de red de apoyo } & \multirow[b]{3}{*}{$F$} \\
\hline & \multicolumn{2}{|c|}{ Tipo 1} & \multicolumn{2}{|c|}{ Tipo 2} & \multicolumn{2}{|c|}{ Tipo 3} & \multicolumn{2}{|c|}{ Tipo 4} & \\
\hline & $\bar{x}$ & $S D$ & $\bar{x}$ & $S D$ & $\bar{x}$ & $S D$ & $\bar{x}$ & $S D$ & \\
\hline \multirow[t]{2}{*}{ Número de familiares en España ........................... } & 5,22 & 5,39 & 1,40 & 2,02 & 1,41 & 2,17 & 1,74 & 2,28 & $21,85^{* * * *}$ \\
\hline & $N$ & $\%$ & $N$ & $\%$ & $N$ & $\%$ & $N$ & $\%$ & $X^{2}$ \\
\hline \multicolumn{10}{|l|}{ Situación en la cadena migratoria } \\
\hline 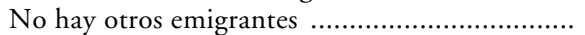 & 4 & 11,4 & 13 & 28,9 & 57 & $31,5^{*}$ & 61 & $20,1^{*}$ & $14,33^{*}$ \\
\hline Antes salieron otros & 22 & 62,9 & 25 & 55,6 & 94 & $51,9^{*}$ & 196 & $64,7^{*}$ & \\
\hline 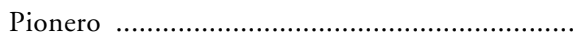 & 9 & 25,7 & 7 & 15,6 & 30 & 16,6 & 46 & 15,2 & \\
\hline \multicolumn{10}{|l|}{ Número de emigrantes en la familia } \\
\hline 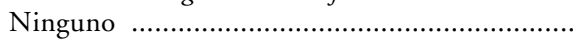 & 4 & 11,4 & 13 & 29,5 & 60 & $33,5^{*}$ & 62 & $20,5^{*}$ & $38,05^{* * * *}$ \\
\hline 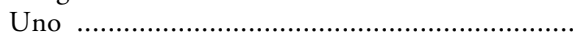 & 5 & 14,3 & 4 & 9,1 & 46 & $25,7^{*}$ & 56 & 18,5 & \\
\hline Dos …1. & 2 & 5,7 & 6 & 13,6 & 14 & $7,8^{*}$ & 56 & $18,5^{*}$ & \\
\hline 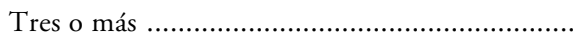 & 24 & $68,6^{*}$ & 21 & 47,7 & 59 & $33,0^{*}$ & 129 & 42,6 & \\
\hline \multicolumn{10}{|l|}{ Residencia de otros emigrantes familiares } \\
\hline Еspaña …… & 18 & $58,1^{*}$ & 9 & 28,1 & 46 & 38,0 & 89 & 36,3 & 6,92 \\
\hline 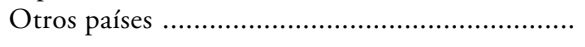 & 13 & $41,9^{*}$ & 23 & 71,9 & 75 & 62,0 & 156 & 63,7 & \\
\hline \multicolumn{10}{|l|}{ Traer a la familia } \\
\hline Sí & 9 & 25,7 & 21 & 46,7 & 66 & 36,3 & 123 & 41,3 & $15,68^{*}$ \\
\hline No & 15 & 42,9 & 19 & 42,2 & 97 & 53,3 & 141 & 47,3 & \\
\hline 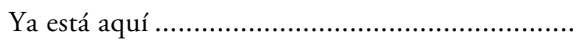 & 11 & $31,4^{*}$ & 5 & 11,1 & 19 & 10,4 & 34 & 11,4 & \\
\hline
\end{tabular}

${ }^{*} \mathrm{p}<0,05 ;{ }^{* *} \mathrm{p}<0,01 ;{ }^{* * *} \mathrm{p}<0,005 ;{ }^{* * * *} \mathrm{p}<0,001$.

FUENTE: Encuesta sobre la integración social de los inmigrantes africanos en Andalucía, 1995. Elaboración propia. 


\section{BIENESTAR PSICOLÓGICO Y UTILIZACIÓN DE RECURSOS SOCIALES} POR TIPO DE RED

Cada tipo de red se asocia a un patrón diferenciado de utilización de los recursos a la hora de afrontar problemas relacionados con el dinero, el trabajo, la vivienda, la documentación, etc. Así, conforme a lo que podríamos esperar, los individuos con un entorno personal basado en el parentesco son los que recurren con más frecuencia a los familiares - y con menos frecuencia a los amigos_- para problemas relacionados con el trabajo, el dinero, la vivienda, la familia y las relaciones. Justo el esquema inverso se da en las redes mixtas centradas en los amigos, excepto en los problemas que tienen que ver con la familia, donde este mismo grupo suele ser la fuente inicial de ayuda (tabla 5).

Ya que la suficiencia de las redes supone recursos naturales escasos o inadecuados, es habitual que los individuos clasificados en el Tipo 3 utilicen -en mayor medida que el resto- los recursos formales para problemas de dinero o que tengan que ver con las relaciones interpersonales. También es más probable que asuman actitudes y comportamientos de autosuficiencia, así como creencias externalistas para problemas de trabajo, salud, dinero y familia.

De acuerdo con el lugar intermedio que ocupan, los sujetos con pequeñas redes de compatriotas muestran la pauta más heterogénea de utilización de los recursos sociales: comparativamente, es más probable que recurran a amigos para afrontar dificultades familiares y de trabajo, y poco probable que opten por los recursos formales para problemas de vivienda, o muestren autosuficiencia en relación a dificultades familiares o con el dinero.

En aquellas áreas donde predomina la utilización de los recursos formales sobre los naturales — como es el caso de la documentación o la salud- se observan menos diferencias entre conglomerados. De todos modos, es posible constatar que también es significativa la proporción en que los sujetos del cluster Tipo 1 acuden en primera instancia a los parientes para resolver cuestiones de documentación, y los de Tipo 2 a los amigos.

Los diversos indicadores de adaptación subjetiva tienen una asociación más débil con el tipo de red: de hecho, no se encuentra relación del cluster de pertenencia con la valoración de la decisión de emigrar ni con la evolución personal experimentada desde la llegada a España, si bien son los de red mínima quienes expresan en mayor medida que les ha ido mal desde que están en el nuevo país.

Donde sí encontramos relación es entre el tipo de red y la satisfacción con las circunstancias actuales, y son de nuevo los sujetos del Tipo 3 los que están menos satisfechos con sus condiciones de vida (tabla 6).

En la tabla 7 describimos, a modo de resumen, las características de cada tipo de red. 
TABLA 5

Utilización de recursos por tipo de red

\begin{tabular}{|c|c|c|c|c|c|c|c|c|c|c|}
\hline & & \multicolumn{8}{|c|}{ Tipo de red de apoyo } & \multirow[b]{3}{*}{$X^{2}$} \\
\hline & & \multicolumn{2}{|c|}{ Tipo 1} & \multicolumn{2}{|c|}{ Tipo 2} & \multicolumn{2}{|c|}{ Tipo 3} & \multicolumn{2}{|c|}{ Tipo 4} & \\
\hline & & $N$ & $\%$ & $N$ & $\%$ & $N$ & $\%$ & $N$ & $\%$ & \\
\hline \multirow[t]{4}{*}{ Documentación } & 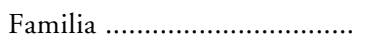 & 5 & $16,7^{*}$ & 0 & 0,0 & 9 & 6,4 & 14 & 5,6 & $18,74^{*}$ \\
\hline & Recursos formales ................. & 22 & 73,3 & 23 & 65,7 & 110 & 78,0 & 200 & 80,0 & \\
\hline & 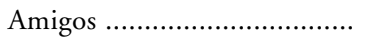 & 3 & 10 & 10 & $28,6^{*}$ & 17 & 12,1 & 32 & 12,8 & \\
\hline & Autosuficiencia ..................... & 0 & 0,0 & 2 & 5,7 & 5 & 3,5 & 4 & 1,6 & \\
\hline \multirow[t]{4}{*}{ Salud } & 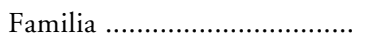 & 4 & 11,8 & 1 & 2,3 & 5 & 2,9 & 20 & 6,8 & 15,55 \\
\hline & Recursos formales ................. & 29 & 85,3 & 38 & 88,4 & 153 & 88,4 & 256 & 86,8 & \\
\hline & Amigos ....................................... & 0 & 0,0 & 4 & 9,3 & 9 & 5,2 & 17 & 5,8 & \\
\hline & Autosuficiencia ..................... & 1 & 2,9 & 0 & 0,0 & 6 & $3,5^{*}$ & 2 & 0,7 & \\
\hline \multirow[t]{4}{*}{ Trabajo } & 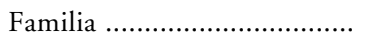 & 13 & $52,0^{*}$ & 0 & $0,0^{*}$ & 11 & 9,2 & 19 & 8,7 & $64,03^{* * * *}$ \\
\hline & Otros & 0 & 0,0 & 1 & 2,9 & 5 & 4,2 & 4 & 1,8 & \\
\hline & Amigos ..................................... & 6 & $24,0^{*}$ & 26 & $76,5^{*}$ & 57 & $47,5^{*}$ & 141 & $24,8^{*}$ & \\
\hline & Autosuficiencia ..................... & 6 & 24,0 & 7 & 20,6 & 47 & $39,2^{*}$ & 54 & 64,7 & \\
\hline \multirow[t]{4}{*}{ Dinero } & 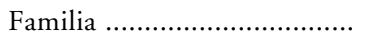 & 18 & $75,0^{*}$ & 6 & $14,3^{*}$ & 50 & 34,2 & 105 & 39,2 & $45,48^{* * * *}$ \\
\hline & Recursos formales ................. & 0 & 0,0 & 2 & 4,8 & 12 & $8,2^{*}$ & 8 & 3,0 & \\
\hline & 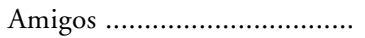 & 4 & $16,7^{*}$ & 32 & $76,2^{*}$ & 67 & 45,9 & 144 & 53,7 & \\
\hline & Autosuficiencia .................... & 2 & 8,3 & 1 & 2,4 & 15 & $10,3^{*}$ & 8 & $3,0^{*}$ & \\
\hline \multirow[t]{3}{*}{ Vivienda } & 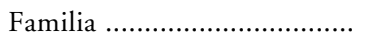 & 17 & $70,8^{*}$ & 3 & $9,4^{*}$ & 29 & 24,6 & 75 & 31,1 & $34,72^{* * * *}$ \\
\hline & Recursos formales ................. & 4 & 16,7 & 6 & 18,8 & 32 & $27,1^{*}$ & 38 & $15,8^{*}$ & \\
\hline & Amigos ................................... & 3 & $12,5^{*}$ & 23 & $71,9^{*}$ & 57 & 48,3 & 128 & 53,1 & \\
\hline \multirow[t]{4}{*}{ Familia } & 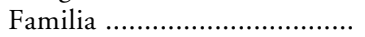 & 27 & $90,0^{*}$ & 14 & 53,8 & 79 & 64,8 & 155 & 65,4 & $22,78^{* *}$ \\
\hline & Recursos formales ................. & 0 & 0,0 & 2 & 7,7 & 9 & 7,4 & 15 & 6,3 & \\
\hline & Amigos $\ldots \ldots \ldots \ldots \ldots \ldots \ldots \ldots \ldots \ldots \ldots$ & 0 & $0,0^{*}$ & 8 & 30,8 & 18 & 14,8 & 55 & $23,2^{*}$ & \\
\hline & Autosuficiencia ................... & 3 & 10,0 & 2 & 7,7 & 16 & $13,1^{*}$ & 12 & $5,1^{*}$ & \\
\hline \multirow[t]{3}{*}{ Relaciones } & 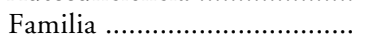 & 13 & $72,2^{*}$ & 0 & $0,0^{*}$ & 19 & 29,7 & 44 & 28,4 & $31,74^{* * * *}$ \\
\hline & Recursos formales ................. & 0 & 0,0 & 1 & 5,6 & 13 & $20,3^{*}$ & 15 & 9,7 & \\
\hline & 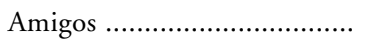 & 5 & $27,8^{*}$ & 17 & $94,4^{*}$ & 32 & 50,0 & 96 & 61,9 & \\
\hline
\end{tabular}

* $\mathrm{p}<0,05 ;{ }^{* *} \mathrm{p}<0,01 ;{ }^{* * *} \mathrm{p}<0,005 ;{ }^{* * * *} \mathrm{p}<0,001$.

FUENTE: Encuesta sobre la integración social de los inmigrantes africanos en Andalucía, 1995. Elaboración propia. 
TABLA 6

Bienestar percibido por tipo de red

\begin{tabular}{|c|c|c|c|c|c|c|c|c|c|}
\hline & \multicolumn{9}{|c|}{ Tipo de red de apoyo } \\
\hline & \multicolumn{2}{|c|}{ Tipo 1} & \multicolumn{2}{|c|}{ Tipo 2} & \multicolumn{2}{|c|}{ Tipo 3} & \multicolumn{2}{|c|}{ Tipo 4} & \multirow[b]{2}{*}{$X^{2}$} \\
\hline & $N$ & $\%$ & $N$ & $\%$ & $N$ & $\%$ & $N$ & $\%$ & \\
\hline \multicolumn{10}{|l|}{ Satisfacción con las circunstancias actuales } \\
\hline 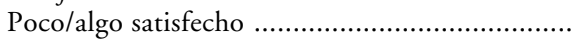 & 20 & 58,8 & 23 & 51,1 & 128 & $69,6^{*}$ & 175 & 57,4 & $9,26^{*}$ \\
\hline 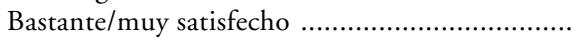 & 14 & 41,2 & 22 & 48,9 & 56 & $30,4^{*}$ & 130 & 42,6 & \\
\hline \multicolumn{10}{|l|}{ Evolución desde la llegada } \\
\hline 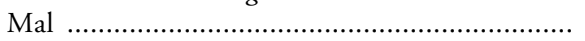 & 6 & 17,6 & 4 & 8,9 & 36 & $19,6^{*}$ & 41 & 13,4 & 7,17 \\
\hline 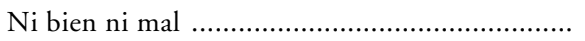 & 11 & 32,4 & 19 & 42,2 & 79 & 42,9 & 135 & 44,1 & \\
\hline 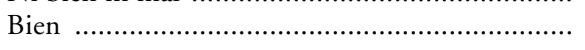 & 17 & 50,0 & 22 & 48,9 & 69 & 37,5 & 130 & 42,5 & \\
\hline \multicolumn{10}{|l|}{ Decisión de emigrar acertada } \\
\hline 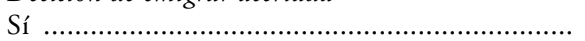 & 27 & 79,4 & 35 & 77,8 & 131 & 73,6 & 239 & 79,1 & 2,06 \\
\hline No & 7 & 20,6 & 10 & 22,2 & 47 & 26,4 & 63 & 20,9 & \\
\hline
\end{tabular}

${ }^{*} \mathrm{p}<0,05 ;{ }^{* *} \mathrm{p}<0,01 ;{ }^{* * *} \mathrm{p}<0,005 ;{ }^{* * * *} \mathrm{p}<0,001$.

FUENTE: Encuesta sobre la integración social de los inmigrantes africanos en Andalucía, 1995. Elaboración propia 


\section{Descripción de los tipos de redes personales}

Tipo

Redes pequeñas con predominio de amigos compatriotas amigos compatriotas

\section{Descripción}

Centran sus solicitudes de ayuda en una pequeña red formada por otros inmigrantes compatriotas: cuentan con unos cuatro vínculos, dos tercios de los cuales son amigos. El uso intensivo de esta red de iguales garantiza una adecuada satisfacción de las diversas necesidades: esto es, las múltiples funciones cubiertas por los amigos permiten a los encuestados no sólo sentirse satisfechos con la ayuda recibida, sino disponer de recursos suficientes para la adaptación subjetiva. Cuentan con un alto índice de antecedentes migratorios en la familia.

La red personal está afectada por la migración, de forma que apenas se cuenta con dos personas para todas las áreas de apoyo. Esto hace que la necesidades estén cubiertas de forma deficiente y que los sujetos se muestren menos satisfechos con sus intercambios de apoyo que el resto. Es poco probable que los miembros de este cluster cuenten con otros emigrantes en la familia.

Redes mixtas de tamaño medio centradas en los amigos

Aunque es típico de la mayoría de los inmigrantes centrar diversos tipos de demandas en el grupo de amigos, sólo un pequeño porcentaje de ellos llega a desarrollar una red de tamaño mediano basada prioritariamente en esta composición. En este crecimiento de la red por medio de la incorporación de nuevos vínculos parece tener un papel importante la relación con españoles, pues es el tipo de red donde tiene más peso tal subgrupo.

Redes de tamaño medio centradas en la familia
Concentran las demandas de apoyo en la familia, hasta el punto de que, en un entorno de unas seis personas, apenas cuentan con no familiares ni con españoles. Esto es posible porque se trata del grupo con más familiares residentes en España y donde son más numerosos aquellos que ya han completado la reagrupación familiar. Es el tipo de red donde menos funciones cumplen los amigos y, al contrario, donde más multiplicidad tienen los vínculos familiares.
Utilización de recursos y bienestar

Siguen una pauta heterogénea de uso de los recursos: acuden significativamente a los amigos para pedir ayuda sobre el trabajo o las relaciones y, comparativamente, no es frecuente que soliciten ayuda a los recursos formales para problemas de vivienda, ni que se muestren autosuficientes en lo que se refiere a la familia o al dinero.

Dado que no cuentan con recursos naturales suficientes, es más común que acudan a los recursos formales para resolver problemas interpersonales o monetarios; o bien que desarrollen actitudes de autosuficiencia e inhibición de las propias necesidades en las áreas de trabajo, salud, dinero y familia. Es el grupo donde podemos encontrar más encuestados que afirmen que les ha ido mal desde que están en España o que se muestren insatisfechos con sus circunstancias actuales.

En consonancia con la composición de su red, recurren significativamente más a los amigos y menos a los familiares cuando experimentan dificultades relacionadas con el trabajo, el dinero, la vivienda o las relaciones personales.

Al contrario del tipo de red anterior, recurren con más frecuencia a familiares y menos a amigos para problemas de trabajo, dinero, vivienda, familia y relaciones. 
Tipo dependiente de la familia local Red pequeña compuesta casi enteramente de lazos familiares locales cercanos.

Tipo integrado localmente Incluye lazos con la familia, amigos y vecinos, e implicación activa con la comunidad.

Tipo local reservado

Tiene algún contacto con parientes, pero está más centrado en los vecinos, con poca implicación comunitaria.

Tipo centrado en la comunidad más amplia

Redes de inmigrantes retirados de clase media, que mantienen gran número de lazos con amigos locales, vecinos y organizaciones voluntarias.

Tipo restringido privado Ancianos solos con escaso contacto social.

\section{Redes familiares de tamaño medio}

Concentran las demandas de apoyo en la familia, hasta el punto de que, en un entorno de unas seis personas, apenas cuentan con no familiares $\mathrm{n}$ con españoles.

Esto es posible porque se trata del grupo con más familiares residentes en España y donde son más numerosos aquellos que ya han completado la reagrupación familiar.

Es el tipo de red donde menos funciones cumplen los amigos y, al contrario, donde más multiplicidad tienen los vínculos familiares.
Redes familiares intensas

Compuesta casi enteramente de miembros de la familia nuclear, es la red más pequeña e íntima, con mayor duración y mayor frecuencia de contacto. Con frecuencia comparten la misma residencia. El potencial de solidaridad es alto.

\section{Redes de parientes}

Red basada en la familia extensa, más amplia que la media. Menos frecuencia de contacto que en la anterior.

\section{Redes pequeñas con predominio de amigos compatriotas}

Centran sus solicitudes de ayuda en una pequeña red formada por otros inmigrantes compatriotas: cuentan con unos cuatro vínculos, dos tercios de los cuales son amigos. El uso intensivo de esta red de iguales garantiza una adecuada satisfacción de las necesidades: las múltiples funciones cubiertas por los amigos permiten a los sujetos no sólo sentirse satisfechos con la ayuda recibida, sino disponer de recursos suficientes para la adaptación.

\section{Redes mixtas de tamaño medio centradas en los amigos} Centrando sus demandas en el grupo de amigos, llegan a desarrollar un red de tamaño mediano basada prioritariamente en dicha composición. En este crecimiento de la red por medio de la incorporación de nuevos vínculos tiene un papel importante un subgrupo de españoles.
Red centrada en los amigos

Es más pequeña que la media y está compuesta mayoritariamente por amigos. A veces se mantienen sólo con contactos ocasionales.

\section{Redes de lazos difusos}

Es la de tamaño más grande, pero también la menos íntima y con menor frecuencia de contactos. Los miembros pueden ser amigos o vecinos, pero los intercambios no son muy significativos.

La red personal está afectada por la migración, de forma que apenas se cuenta con dos personas para todas las áreas de apoyo: las necesidades están cubiertas de forma deficiente y los sujetos se muestran poco satisfechos con sus intercambios de apoyo. Es poco probable que en este cluster cuenten con otros emigrantes en la familia. 


\section{DISCUSIÓN}

Para discutir nuestros resultados compararemos nuestra tipología con otras clasificaciones elaboradas con antelación y valoraremos las diferencias observadas entre las cuatro categorías en la funcionalidad de la red, la utilización de recursos sociales y el bienestar psicológico, además de en otras variables psicosociales.

A continuación, revisamos las similitudes y divergencias de esta clasificación con las elaboradas por Wenger (1991) y Litwin (1995). Aunque las tres tipologías difieren en las variables criterio y en el tipo de población, nos parece que la comparación de las mismas es una vía útil para iniciar la discusión.

En las tres clasificaciones parece que las dimensiones fundamentales en la determinación de los tipos de redes son el tamaño y la composición (y particularmente la proporción de familiares y no familiares en la red). En el caso de los inmigrantes africanos, es singular el hecho de que sólo en una de las cuatro clases predominen los vínculos familiares, mientras que en las otras tipologías al menos dos categorías están formadas mayoritariamente por parientes. Esta divergencia podemos atribuirla en parte a las diversas poblaciones de las que se ha derivado cada clasificación, ya que el desplazamiento internacional experimentado por los participantes en nuestro estudio supone la ruptura - al menos temporalmente- con los vínculos familiares, y, en consecuencia, recurrir al grupo de iguales disponible - generalmente otros compatriotas emigrantes - es la primera y más habitual vía de reconstrucción de la red. Si bien el examen de Litwin también se realizó con inmigrantes rusos en Israel, consistió en una muestra de ancianos, por lo que es más probable que contasen con antecedentes familiares en el país de destino (Angel y Angel, 1992; Bialik-Gilad, 1988; Die y Seelbach, 1988; Ikels, 1986) y, por tanto, que entrasen a formar parte desde el primer momento de reasentamiento de sus redes sociales.

Además de esta observación de carácter general, es posible señalar coincidencias de categorías específicas. En primer lugar, las redes de tamaño medio centradas en la familia, observadas en los inmigrantes africanos en Andalucía, son comparables a la red dependiente de la familia local de Wenger o a la red familiar intensa de Litwin. En los tres casos se trata de estructuras sociales compuestas casi enteramente por lazos de la familia nuclear, con los que se comparte residencia, y que desarrollan intercambios de naturaleza íntima. Sin embargo, en nuestro estudio el tamaño es ligeramente superior a los dos referentes de comparación: en todo caso, esto no la convierte en una red de familia extensa, en la terminología de Litwin, ni llega a ser del tipo integrado localmente que, de acuerdo con Wenger, contiene lazos con amigos y vecinos y se implica activamente con la comunidad.

En el presente estudio, la red centrada en la familia tiene por término medio tres años más de residencia que el resto, lo que sugiere un patrón evolutivo de formación de la estructura de apoyo en el que el reagrupamiento 
familiar tiene un papel determinante. Además, suponemos que, con más tiempo de estancia en España, los inmigrantes podrán desarrollar redes más amplias de familia extensa - como las observadas en el estudio de Litwin- o bien completar su cúmulo de proveedores con más españoles y, así, conformar redes integradas localmente — al modo de las descritas en el trabajo de Wenger-.

En segundo lugar, tanto la clasificación de Wenger como la de Litwin incluyen dos tipos de estructura reticular, en los que predominan las relaciones no basadas en el parentesco, que son equiparables a las redes pequeñas con predominio de compatriotas y las redes mixtas de tamaño medio centradas en los amigos, de los inmigrantes residentes en Andalucía. Atendiendo a la composición, Litwin (1995) considera la red focalizada en los amigos similar a aquella centrada en la comunidad más amplia (de Wenger), y la de lazos difusos parecida al tipo local reservado, de Wenger. Sin embargo, si además tenemos en cuenta el tamaño de la red, podríamos realizar la comparación en sentido inverso al que plantea Litwin y establecer dos categorías: una que puede contar con algún contacto con parientes, pero está compuesta sobre todo por un pequeño grupo de amigos, conocidos o vecinos (que sería la red reservada local según Wenger, centrada en los amigos para Litwin, y red pequeña con predominio de amigos compatriotas en nuestra terminología); y otra que mantiene el mayor número de lazos con no familiares (que sería la red centrada en la comunidad más amplia según Wenger, de lazos difusos para Litwin, y red mixta de tamaño medio centrada en los amigos en nuestra terminología).

Ambas categorías evidencian que una pequeña red de amigos puede ser una vía adecuada para sustituir temporalmente a la familia, y que potenciar los vínculos no familiares - incorporando, entre otros, miembros de la sociedad receptora - es un medio, alternativo al reagrupamiento, útil para construir una red de tamaño medio, habitualmente en menos tiempo del que supone la reubicación del núcleo familiar. Uno y otro procedimiento revelan la trascendencia de la amistad en el proceso de adaptación de los inmigrantes.

Por último, la red mínima con predominio de amigos compatriotas, observada entre los inmigrantes residentes en Andalucía, es muy similar a la red limitada privada de Wenger: si en la primera apenas se cuenta con compañeros que puedan cubrir las necesidades de la persona focal, en la segunda se hace referencia a los ancianos que viven solos y tienen escaso contacto social. Aunque la tipología de Litwin no cuente con una clase de estas características, hay cinco sujetos que no fueron clasificados en la tipología y bien podrían compartir los rasgos anteriores: de hecho, tres de ellos no disponían de ningún contacto social, y los otros dos contaban con uno y tres vínculos solamente.

Otra evidencia de nuestro análisis es el impacto de la inmigración en las redes de apoyo social, que viene a sumarse al estudio de Harper (1987) con 
ancianos escoceses, en el que mostró —utilizando procedimientos etnográficos- que la movilidad residencial en sí misma tiene efectos importantes en la composición y la frecuencia de contacto con los proveedores de apoyo. Este autor distinguió tres tipos de ancianos en función de su estructura de ayuda: en primer lugar, aquellos que no se han desplazado de su lugar de residencia habitual ubican en los familiares todas las demandas de apoyo - tanto diario como de crisis, tanto físico como psicológico-; frente a ellos, los ancianos que cambian de residencia al jubilarse recurren a la comunidad más amplia —-formal e informal— cuando no disponen de la familia en el nuevo contexto; mientras que, por último, quienes al desplazarse cuentan con familiares en el ámbito local recurren a la familia para la obtención de ayuda física y psicológica, pero también acuden a la comunidad más amplia para la ayuda física.

A pesar de que tamaño y composición aparecen como elementos clave en la determinación de la clase de red que se posee, la tipología también revela diferencias significativas en las funciones de apoyo: de esta manera, se observan divergencias en la disponibilidad, la utilización y la suficiencia de la ayuda, así como en la satisfacción con los intercambios de apoyo y la multiplicidad de los proveedores. Parte de esas diferencias en los niveles de apoyo parecen venir condicionadas precisamente por el tamaño y la composición de la red. De este modo, los tres mismos escalones que podemos establecer en relación al tamaño se repiten en las discrepancias de apoyo emocional disponible, apoyo tangible disponible y apoyo tangible utilizado. Igualmente, las redes que peor cubren las necesidades de los inmigrantes son también las de menor tamaño. De hecho, en la literatura sobre el tema se ha documentado que el escaso número de relaciones personales observadas en algunos inmigrantes afecta tanto a la cantidad como a la calidad de los intercambios de ayuda (Leslie, 1992; Lynam, 1985; Patel, 1992): la escasez de contactos conduce a que se sobrecargue a los pocos individuos disponibles o a que aumente la frecuencia de conflictos, así como las crisis individuales o interpersonales (Sluzki, 1992). En nuestro caso, los entrevistados asignados al tercer agregado no sólo creen insuficiente su entorno personal, sino que están poco satisfechos con los intercambios de ayuda experimentados.

Por otro lado, entre las redes más amplias (Tipos 1 y 2) la composición nacional podría estar generando diferencias, ya que aquellos que cuentan con una mayor proporción de españoles en su entorno personal disponen de más recursos informativos y utilizan más soporte informativo y emocional que aquellos que están rodeados sobre todo por parientes y, por tanto, connacionales. En ese sentido, otros autores coinciden en atribuir a los miembros de la sociedad de acogida un rol valioso como proveedores de información (Aroian, 1992; Lynam, 1985), pero nuestros datos no nos permiten ir más allá de la mera conjetura al respecto.

También es posible recurrir a la composición para explicar el número de funciones de apoyo que cumplen los vínculos familiares y no familiares: parece 
existir una relación inversa entre la multiplicidad de los amigos y la multiplicidad de padres, hermanos, hijos o incluso la pareja. En otras palabras, los vínculos familiares proporcionan más tipos de ayuda a los participantes con redes centradas en los parientes que al resto, mientras que con los amigos ocurre lo contrario. Es de suponer que los iguales sustituyen las funciones que cumplía la familia antes de la emigración, en un esfuerzo de adaptación al nuevo contexto, y que tales competencias son restituidas a la parentela después del reagrupamiento. Estos resultados coinciden con las apreciaciones de Harper (1987), que observó que la presencia de la familia en el entorno personal de los ancianos disminuye el número de amigos no parientes, en la medida en que la disponibilidad de familiares para la atención de necesidades sociales y de apoyo reducen la demanda y el tiempo disponible para los amigos.

Una excepción a la regla la constituye el hecho de que la satisfacción con los intercambios de ayuda no varía en función de la tipología de red. Es posible presumir que la mayoría de los miembros de la red comparten con la persona focal la misma carencia de recursos materiales y afrontan dificultades económicas semejantes, con lo que resulta difícil satisfacer los requerimientos de ayuda tangible (con independencia del tipo de red de que se disponga). Este extremo fue descrito por Aroian (1992), que observó, en una muestra de inmigrantes polacos recién llegados a Estados Unidos, que la fuente principal de ayuda tangible eran compatriotas de oleadas migratorias anteriores, pues aquellos con escaso tiempo de estancia experimentaban su misma carencia de recursos materiales.

Del mismo modo que varían las funciones de apoyo, de cada tipo de red se derivan unos patrones de utilización de los recursos sociales bien diferenciados: en consonancia con la composición de su entorno personal, unos individuos recurrirán más a los amigos (Tipo 2) y otros más a la familia (Tipo 1), y aquellos que no cuentan con recursos naturales suficientes tendrán que confiar en los servicios formales para determinados problemas, o bien desarrollar actitudes de autosuficiencia e inhibición de las propias necesidades (Tipo 3). De acuerdo con las conclusiones de la literatura al respecto, la norma general de acudir en primer lugar a los grupos naturales para solicitar ayuda sólo se rompe o bien por la especificidad del problema —salud, documentación, educación- o bien por la insuficiencia de las redes naturales (Die y Seelbach, 1988; Griffith, 1984; Griffith y Villavicencio, 1985; Ikels, 1986; Leslie, 1992; Lynam, 1985; Patel, 1992; Raymond, Rhoads y Raymond, 1980; Zhang, Snowden y Sue,1998). En un estudio representativo de los africanos residentes en Andalucía, comprobamos que la fuente inicial de ayuda para la mayoría de los inmigrantes eran los amigos, seguidos, por motivos de composición de la red, por la familia. Por su parte, la administración era una fuente específica de ayuda para temas de salud, documentación y educación, mientras que los encuestados recurrían a organizaciones no gubernamentales para obtener atención jurídica, así como cursos de formación y de español (Martínez et al., 1996). 
Los cuatro conglomerados se diferencian, además, en aspectos sociales. Particularmente, la posición que el individuo ocupa en la cadena migratoria está directamente vinculada al tipo de red de apoyo que puede desarrollar una vez en el país de destino: así, quienes tienen más familiares residentes en España, y aquellos que ya han completado la reagrupación, gozan de más probabilidades de focalizar sus demandas de apoyo en los parientes; y, al contrario, no tener otros emigrantes en la familia se relaciona con la conformación de redes personales mínimas. En otras palabras, el potencial de crecimiento y estructuración de las relaciones personales de los inmigrantes a nivel individual viene condicionado por el grado de desarrollo de las cadenas migratorias a nivel colectivo. Si prácticamente en el 94 por 100 del colectivo advertimos el predomino de amigos, no es sólo - tal y como ya hemos mencionado- como resultado de una estrategia de sustitución temporal de la familia asociada a la emigración, sino que también obedece al carácter reciente de este fenómeno en el contexto receptor. Es de suponer que, en la medida en que se consoliden en Andalucía comunidades de inmigrantes más amplias, estables y estructuradas, será más probable el desarrollo de redes personales centradas en la familia, o con una composición más equilibrada de parientes y no parientes. Y, a la inversa, las redes personales mínimas representarán cada vez una proporción más pequeña de la población. En ese sentido, Angel y Angel (1992) demostraron en un estudio con ancianos mexicanos, cubanos y puertorriqueños inmigrantes en Estados Unidos que el desplazamiento a enclaves étnicos permite reemplazar con facilidad los lazos sociales de origen y disminuye el riesgo de enfermedad. Por consiguiente, emigrar solo a un nuevo país con escasos contactos es potencialmente más estresante que emigrar con la familia a un enclave étnico bien establecido, como el que conforman los cubano-americanos en Florida o los mexicanos en Los Angeles. En otro trabajo -incidiendo en el mismo tema-, Griffith (1984) observó que la segunda y tercera generación de mexicanos tenían redes de apoyo más amplias y más integradas que los de primera generación.

En un estudio amplio con diferentes colectivos inmigrantes en la Comunidad Autónoma de Madrid también se ha documentado la conformación de redes basadas mayoritariamente en criterios nacionales y étnicos, así como el impacto de las mismas en el traslado a España, la obtención de alojamiento y el acceso al trabajo (Giménez, 1993).

En definitiva, las redes de apoyo social de los inmigrantes informan adecuadamente del progreso de su adaptación personal al nuevo contexto, y se relacionan de forma directa con la utilización de servicios y con el bienestar psicológico. Por otro lado, la clasificación de las redes de apoyo parece una herramienta útil para identificar cuáles son los elementos que permanecen en el fenómeno de continuidad de los intercambios de ayuda que ha sido descrito por el modelo de convoy social (Kahn y Antonucci, 1980). Además, la tipología ensayada permite distinguir a aquellos que cuentan con recursos naturales insuficientes de otros sujetos con más éxito a la hora de garantizarse un soporte 
afectivo e instrumental mínimo; y, en segundo lugar, pone de manifiesto la relevancia del reagrupamiento familiar en la conformación de un sistema de ayuda equilibrado y suficiente. Por todo ello, el tipo de red de apoyo puede considerarse un indicador psicosocial útil tanto en estudios con fines descriptivos como en análisis de carácter prospectivo: en efecto, no posibilita al entrevistado conjeturar los objetivos de evaluación del investigador, a la vez que sirve para resumir información valiosa sobre las condiciones sociales de vida del individuo.

\section{REFERENCIAS BIBLIOGRÁFICAS}

ADAMS, R.G. (1986): «Secondary friendship network and psichological well-being among ederly woman", Activities, Adaptation and Aging, 8 (2): 59-72.

ANGEL, J. L., y ANGEL, R. J. (1992): «Age at migration, social connections, and well-being among elderly Hispanics», Journal of Aging and Health, vol. 4 (4), noviembre: 480-499.

Aroian, K. J. (1992): «Sources of Social Support and Conflict for Polish Immigrants», Qualitative Health Research, vol. 2, núm. 12, mayo: 178-207.

BARRERA, M. (1980): "A method for the assessment of social support networks in community survey research", Connections, 3: 8-13.

BERrY, J. W. (1997): «Inmigration, acculturation and adaptation», Applied Psychology: an International Review, 46, 1: 5-34.

BIALIK-GILAD, R. (1988): «The effects of immigration on social support networks of older salvadorans», Dissertation Abstracts International, 51, 1, julio: 293-A.

Bотт, E. (1990): Familia y red social, Taurus, Madrid.

BoyD, M. (1989): «Family and Personal Networks in International Migration: Recent Developments And New Agendas», International Migration Review, 23, núm. 13: 638-670.

CAPLAN, G. (1974): Support systems and community mental health: lectures on concept development, Behavioral Publications, New York.

CASSEL, J. (1974): «Psychosocial process and stress: theoretical formulations», International Journal of Health Services, 4: 471-482.

CiAfARDO, E. O. (1991): "Cadenas migratorias e inmigración italiana. Reflexiones a partir de la correspondencia de dos inmigrantes italianos en Argentina, 1921-1938», Studi Emigrazione/Etudes Migrations, 28, 102, junio: 233-256.

Clarke, D., y Jensen, M. (1997): "The effects of social support, life events, and demographic factors on depression among Maori and Europeans in New Zealand rural, town, and urban environments», Journal of Community Psychology, 25 (4): 303-323.

Coвb, S. (1976): «Social support as a moderator of the life stress», Psychosomatic Medicine, 38 : 300-314.

Die, A. H., y Seelbach, W. C. (1988): «Problems, sources of assistance, and knowledge of services among elderly vietnamese immigrants", The Gerontological Society of America, vol. 28, núm. 14: 448-452.

Furnham, A., y LI, Y. H. (1993): «The psychological adjustment of the Chinese community in Britain: A study of two generations», British Journal of Psychiatry, vol. 162, enero: 109-113.

GimÉnEZ, C. (1992): Madrid y el desafio de la inmigración, Consejería de Integración Social de la Comunidad Autónoma de Madrid, Madrid.

- (1993): Inmigrantes extranjeros en Madrid, Consejería de Integración Social de la Comunidad Autónoma de Madrid, Madrid.

Golding, M. J., y Baezconde-Garbanati, L. A. (1990): «Ethnicity, Culture and Social Resources», American Journal of Community Psychology, vol. 18, núm. 13. 
GotTlieb, B. H. (1981): Social Networks and social support, Sage, Beverly Hills.

Granovetter, M. S. (1973): «The strength of weak ties», American Journal of Sociology, 78: 1360-1380.

Gregorio, C. (1998): Migración femenina. Su impacto en las relaciones de género, Narcea de Ediciones, Madrid.

GrifFITH, J. (1984): «Emotional support providers and psychological distress among Anglo- and Mexican-Americans», Community Mental Health Journal, 20: 182-201.

GRIFFITH, J., y VILLAVICENCIO, S. (1985): «Relationships among acculturation, sociodemographic characteristics and social support in Mexican-American adults", Hispanic Journal of Behavioral Sciences, 7 (1): 75-92.

Gordon, G., y Wenger, G. C. (1993): «Dynamics of support networks: Differences and similarities between vulnerable groups", Irish Journal of Psychology, 14 (1): 79-98.

Guimon, J.; Ruiz, A.; Apodaca, P.; De Ros, P., y Sota, E. (1985): «Red social en la población de Guecho", Psiquis, vol. 6 (4): 121-130.

HAMMER, M.; MAKIESKY BARROW, S., y GUTWIRTH, L. (1978): «Social network and Schizophrenia», Schizophrenia Bulletin, vol. 4 (4): 522-544.

HARPER, S. (1987): "The kinship network of the rural aged: a comparison of the indigenous elderly and the retired immigrant", Ageing and Society, 7, 3, septiembre: 303-327.

IKELS, C. (1986): «Older Immigrants and Natural Helpers», Journal of Cross-Cultural Geronto$\log y, 1,2: 209-222$.

IOÉ (1987): «Los inmigrantes en España», Documentación Social, núm. 66.

IZQUIERDO, A. (1994): Cuestionario sobre la integración social de los marroquies en Murcia, policopiado.

- (1995): Los inmigrantes extranjeros. Perfil sociodemográfico, Junta de Andalucía, Sevilla.

JANES, C. R. (1990): «Migration, changing gender roles and stress: The Samoan case», Medical Anthropology, vol. 12 (2), marzo: 217-248.

KAHN, R. L., y ANTONUCCI, T. C. (1980): «Convoys over the life course: Attachment, roles and social support», en P. Baltes y O. Brim (eds.), Life span development and behavior, vol. 3, Academic Press, San Diego (CA), pp. 253-286.

KUO, W. H., y TSAI, Y. M. (1986): «Social networking, hardiness and immigrant's mental health", Journal of Health and Social Behavior, vol. 27 (2), junio: 133-149.

LESLIE, L. A. (1992): "The role of informal support networks in the adjustment of Central American immigrant families», Journal of Community Psychology, vol. 20 (3), julio: 243-256.

LiTWIN, H. (1995): "The social networks of elderly immigrants: An analytic typology», Journal of Aging Studies, vol. 9 (2), verano: 155-174.

- (1997): «Support networks type and Health Service utilization», Research on Aging, vol. 19 (3): 274-299.

- (1999): «Support network type and patterns of help giving and receiving among older people», Journal of Social Service Research, 24 (3-4): 83-101.

LYNAM, M. J. (1985): "Support networks developed by immigrant women», Social Science and Medicine, vol. 21 (3): 327-333.

Martínez, M. F.; GARCía, M., y MAYA, I. (1999): «El papel de los recursos sociales naturales en el proceso migratorio", Intervención Psicosocial, vol. 8, núm. 12: 221-232.

Martínez, M. F.; García, M.; Maya, I.; Rodríguez, S., y CheCA, F. (1996): La integración social de los inmigrantes africanos en Andalucía. Necesidades y Recursos, Consejería de Asuntos Sociales de la Junta de Andalucía, Sevilla.

MCFarlane, A. H., y otros (1980): "A longitudinal study of the influence of the psychosocial environment on health status: a preliminary report», Journal of Health and Social Behavior, 21: 124-133.

Miller-Loncar, C. L.; Erwin, L. J.; Landry, S. H.; Smith, K. E., y Swank, P. R. (1998): "Characteristics of social support networks of low socioeconomic status african american, anglo american, and mexican american mothers of full-term and preterm infants», Journal of Community Psychology, vol. 26, núm. 12: 131-143. 
Mitchell, J. C. (1969): «The concept and use de social networks», en J. C. Mitchell (ed.), Social Networks in Urban Situation, Manchester University Press, Manchester.

MOSER (1973): «Social indicators: system, methods and problem», The Review of Income and Wealth, 134.

PAtel, N. (1992): "Psychological disturbance, social support and stressors: a community survey of immigrant Asian women and the indigenous population", Counselling-Psychology-Quarterly, vol. 5 (3): 263-276.

RAYMOND, J. S.; RHOADS, D. L., y RAYMOND, R. I. (1980): "The relative impact of family and social involvement on Chicano mental health", American Journal of Community Psychology, 8: 557-569.

REQUENA, F. (1991): Redes sociales y mercado de trabajo, CIS/Siglo XXI, Madrid.

- (1994): Amigos y redes sociales. Elementos para una sociología de la amistad, Siglo XXI Editores, Madrid.

- (1996): Redes sociales y cuestionarios, Cuadernos Metodológicos, núm. 118, Centro de Investigaciones Sociológicas, Madrid.

Rodríguez, J. A. (1995): Análisis estructural y de redes, Cuadernos Metodológicos, núm. 116, Centro de Investigaciones Sociológicas, Madrid.

SLUZKI, C. E. (1992): «Disruption and reconstruction of networks following migration/relocation", Family Systems Medicine, Win, vol. 10 (4): 359-363.

SYCIP, L. M., y FAWCET, J. T. (1988): «Expectations, family networks and emigration: A study of filipino decisión-making», Philippine Journal Psychology, 56-71.

TAFT, R. (1986): "Methodological considerations in the study of immigrant adaptation in Australia", Australian Journal of Psychology, vol. 38 (3), diciembre: 339-346.

Thorts, P. (1982): "Conceptual, Methodological and Theoretical Problems in studying social support as a buffer against stress", Journal of Helath and Social Behavior, 23: 145-149.

Vega, W. A.; Kolody, B.; Valle, R., y Weir, J. (1991): «Social networks, social support, and their relationship to depression among immigrant Mexican women", Human Organization, vol. 50 (2), verano: 154-162.

WENGER, G.C. (1977): «Social networks and the prediction of elderly people at risk», Aging and Mental Health, 1 (4): 311-320.

- (1990): Personal care: variation in network type.

- (1991): "A network tipology: from theory to practice», Journal of Aging Studies, 5: 147-162.

- (1993): «The formation of social networks: Self help, mutual aid, and old people in contemporary Britain», Journal of Aging Studies, 7 (1): 25-40.

Wenger, G. C., y ShahtahmasebI, S. (1991): «Survivors: Support network variation and sources of help in rural communities», Journal of Cross Cultural Gerontology, 6 (1): 41-82.

ZHANG, A.; SNOWDEN, L. R., y SUE, S. (1998): «Differences between asian and white americans' help seeking and utilization patterns in the Los Angeles area», Journal of Community Psychology, vol. 26, núm. 14: 317-326. 


\begin{abstract}
We elaborate a classification of the social support networks of African immigrants in Andalucia. We did a Quick Cluster analisys with several criterion variables: the size, the composition, the availability, the utilization and the sufficiency of the social support network. With a group of 600 African immigrants, we obtained four categories: 1) compatriots friends focused small network; 2) insufficient network; 3) medium size network compound by spanish and compatriots friends, and 4) medium size network conformed by relatives. The tipology serves to distinguish levels of psychological well-being, type of utilization of services and other social, demographic, and psychological characteristics.
\end{abstract}


NOTAS DE INVESTIGACIÓN 